\title{
Bilateral Transmigration of Mandibular Canines-A Case Report and Review of Literature
}

\author{
Dr. Syed Ansar Ahmad ${ }^{1}$, Dr.S.S.Ahmad, ${ }^{2}$ Dr.R.N.Poddar ${ }^{3}$, \\ Dr.Rahnuma masood ${ }^{4}$ \\ ${ }^{I}$ Assistant professor,Deptt. of Oral and Maxillofacial Surgery Faculty of Dentistry, Jamia Millia Islamia,New \\ Delhi,. India \\ ${ }^{2}$ Associate professor and chairman, Department of Oral surgery, Z.A.Dental College, Aligarh. \\ U.P.9411981399, India \\ ${ }^{3}$, Professor and Head, Deptt of Oral and Maxillofacial surgery, R.Ahmed Dental College and Hospital \\ Kolkata.9830516189,India \\ ${ }^{4}$ Private practitioner New Delhi, India
}

\begin{abstract}
Transmigration of mandibular canines is a rare phenomenom. Bilateral transmigration of mandibular canine is even much rarer. Treatment can be orthodontic or surgical. Orthodontic treatment is very cumbersome. Here we are reporting a case of a bilateral transmigration of canines which was treated surgically, with great results. Also we are giving a brief review of literature.
\end{abstract}

Key words; abgel, bilateral, canine, management, transmigration,

\section{Introduction}

Pre -eruptive migration of the tooth across the midline is termed as transmigration. Failure of eruption and intraosseous migration of an impacted tooth is an unusual developmental dental anomaly that dental professionals encounter in their practice. These are particularly significant if the impacted tooth is canine due to an aesthetic and functional reason. The presence of an over-retained mandibular deciduous canine or missing permanent canine indicates tooth to be investigated radio graphically.

It is not uncommon to find over retention of deciduous teeth even after the normal age of eruption which may vary from months to years and sometimes it is for whole life too. Usually it is a rare event; however, third molars and maxillary canines are exceptions. These teeth either may remain impacted in the path of their eruption or they may move towards the midline. When such teeth cross the midline it is termed as dental transmigration. Of course, it is a pre eruptive phenomenon. Pre- eruptive migration of a tooth across the midline is termed transmigration ${ }^{[1]}$. Although Ando et al used the term transmigration first ${ }^{[2]}$, Tarsitano defined it as the phenomenon of an unerupted mandibular canine crossing the midline ${ }^{[3]}$ but it was Javid ${ }^{[1]}$ who expanded the definition to include the cases in which were than half of the tooth had passed through midline. This type of cases have been published in with various terms. This rare anamoly has been used in the literature with various terms like anamolous ${ }^{[4]} 7$, malpositioned ${ }^{[5]}$ displaced tooth ${ }^{[6]}$. According to Joshi the tendency of a canine to cross the barrier of the mandibular midline suture is more important than the distance traveled in the same side where it developed.

We are reporting this case to add one more case in the literature of transmigration and to review the already reported such cases, to prepare the epidemiological characteristics of this anomaly and to add the novelty of this case as Kissing (transmigrated) mandibular canines.

\section{Case Report}

A 12 years old female patient reported to our centre with chief complaint of local discomfort for last one year with no history of any previous trauma. Extraoral examination revealed a well defined, non-tender swelling in the labial vestibule without any regional lymphadenopathy. However, the anterior teeth were firm, non-tender.

Radiological evaluation of occlusal view and OPG revealed the presence of bilateral impacted mandibular canine, apically placed to incisors, horizontally placed, both crossing the midline and half part of both the teeth were overlying but opposite to each other. Thus a diagnosis of transmigrated canine was made. Shift cone technique was employed to arrive at a conclusion that the transmigrated canine is labially placed.

A vestibular incision was given in the anterior region, bone was cut using micromotor and flat fissure bur and window was made. Canine migrating from left side was decapitated first. Then its crown was removed which followed the removal root. More bone was cut and the other tooth was removed. After removal of teeth an osseous cavity was left and it was filled by abgel. So that no dead space remained. The wound was closed by 
interrupted sutures, which was removed after a week. The healing was uneventful and post operative radiographs taken after 3 and 6 months showed substantial amount of formation of bone.

\section{Discussion}

Transmigration, a rare anomaly, occurs 20 times less than the upper canine ${ }^{[1]}$. This clinical condition must be suspected, if the lower canine is absent from the arch or if the canine has not erupted even after passing significant time after the normal age of eruption. In some cases, agenesis of the lateral incisors and lower premolars has been detected. ${ }^{[2]}$

The etiology of transmigration is unknown and many causes have been suggested. Bruzst ${ }^{[5]}$ is of opinion that the canine tooth germ is situated in front of the lower incisors and that facial growth pushes it towards the contralateral side. Other factors may be an abnormally strong eruption force or a change which affects the crypt of the tooth germ might lead to erroneous eruption ${ }^{[7]}$. Other factors favouring the appearance of this anomaly are: premature loss of deciduous teeth, osteodental discrepancy, agenesis of the lower lateral incisors, endocrinopathy and hereditary factors, traumas and odontomas ${ }^{[2]}$. We feel misdirected eruption forces may be also a cause. Although transmigrated canine don't erupt normally but it has been reported that rarely normal eruption is also possible The transmigration begins between 6 to 8 years of age and before the root formation is complete. ${ }^{[12]}$

Clinically cases of transmigrated teeth are symptomless and they are detected when the radiography is done due to one or other reason. Sometimes they cause neurological changes by causing compression of inferior alveolar nerve by the impacted tooth and sometimes they are associated with certain pathological conditions like dentigerous cysts, odontomas and the appearance of other impactions.

Pre-eruptive migration of tooth across the midline is termed as transmigration.. Although this condition has only been reported for mandibular canines, Ayden and Yilmaz ${ }^{[9]}$ reported the first case of maxillary canine transmigration in literature. In 2002, Mupparapu ${ }^{[10]}$ classified the impacted transmigrated canines and divided them in following five types according to their migratory pattern and their position in the jaw:

Type 1:- Canine is impacted mesioangularly across the midline, labial or lingual to the anterior teeth with the crown portion of tooth crossing the midline.

Type 2 :- Canine is horizontally impacted near the inferior border of the mandible below the apices of incisors.. Type 3 :- Canine has erupted either mesial or distal to the opposite canine.

Type 4 :- Canine is horizontally impacted near the inferior border of the mandible below the apices of either premolars or molar on the opposite side.

Type 5 :- Canine is positioned vertically in the midline with the long axis of the tooth crossing the midline.

Mupparapu's type 1 followed by type 2, are the most frequently occurring pattern. Type 4 and 3 occur less frequently, while type 5 is the least frequent ${ }^{[10]}$,However, pattern 2 corresponds to our case (Canine is horizontally impacted near the inferior border of the mandible below the apices of incisors.).

In the literature different report have been documented related to the prevalence, gender, location ( left or right ) maxillary or mandibular transmigrated canines.The incidence of transmigration has been reported to be $0.1 \%$. Impaction of maxillary canines have been reported to occur 20 times more but unlike the mandibular canine, are impacted maxillary canine has never been found migrating across the midpalatal suture to the other side of maxilla except one transmigration reported in a recent publication.

Because tooth is generally impacted in a horizontal position under the apices of the permanent teeth, adjacent to the mandibular border or a palataly impacted canine is sometimes postioned horizontally very high in the palatal vault, close to the floor of nasal cavity therefore an impacted tooth in the maxilla or in the mandible cannot be detected with inspection or palpation in the clinical examination and is usually detected on a routine periapical radiography. Therefore while examining a patient with over retained deciduous canine a probable diagnosis of impacted canine should be considered. Such patient should be investigated radiographically. Whith the advent of panoramic radiography, number of case report that published the prevalence of transmigrated canines are in rise. Migrated canines usually remain impacted and they are asymptomatic or some time they may erupt ectopically at the middle or on the opposite side of the arch. Although most of the transmigrated cases are reported to be unilateral, transmigration may also be bilateral.

The absence of delayed eruption of permanent mandibular canines or the over retention of a primary canine in the each are common clinical findings if an impacted or transmigrated tooth for that reason most of the transmigrated canine are generally asymptomatic. The transmigrated canine should be kept under radiographic observation and radiographs should be taken periodically to observe the movement of this tooth.

Surgical removal, transplantation, radiographic follow up and surgical exposure with orthodontics treatment are suggested treatment options for transmigration canines. If the decision of extraction is taken it should be kept in mind that, although the teeth have transmigrated in the other side of the midline, they still 
maintain their nerve supply from the originating side. That's why it is extremely important to anesthetize. The nerve on the originating side. Transplantation is another option if the mandibular incisors are in normal position and there is enough space for transmigrated canine.

Such transmigrated canines should ideally be treated by interceptive methods. This is a difficult task. For interception, the patients with clinical findings of impacted canine related to transmigration, and between 8 and 9 years of age should undergo a clinical and radiological examination. Diagnosis at this stage will open large number of treatment options like surgical repositioning, autotransplantation or surgical/orthodontic treatment, depending on the individual characteristics of the patient, location and inclination of the tooth ${ }^{[4]}$.In patients under 14 years old, before extracting the tooth, other options should be considered and the case should be carefully assessed. In patients over 14 years old, significant changes are not expected and extraction should only be considered if the patient rejects orthodontic treatment. ${ }^{[2]}$

Once transmigration is established extraction of impacted canine may be a treatment of choice.. Although the tooth is extracted on the contralateral side to the transmigrated canine, the tooth maintains its nervous innervation on the side that the germ is formed. This must be taken into account during the process of tooth extraction. ${ }^{[12]}$

\section{Conclusion}

Treatment option can be surgical or orthodontic. Treatment option in these patients is observation and clinical check ups, with radiographs taken periodically. In the case in question, given the location of the canine, the orthodontist felt it to be more prudent to complete orthodontic treatment using a deciduous canine as a permanent tooth, and given that the canine apices were closed, advised against transplantation and suggested extraction. The patient was informed of all the aspects of his treatment, and possibility of premature loss of the deciduous canine, which would have to be covered with an osseointegrated implant in the said position.

[1] Javid B.R.Transmigration of impacted mandibular cuspids. International journal of oral surgery.volume 14,issue 6,dec1985,pages547-549

[2] Ando S.AizaeaK.Nakashima T.Sanka Y.Shimbo K.Kiyikawa k.Transmigration Process of impacted mandibular cuspid.J Nihon univ sch Dent1964;6;66-71.

[3] Tarsitano JJ,wooten J W,Burditt J T.Transmigration of nonerupted mandibular canines.report of cases.J Am Dent association $1971 ; 82 ; 1395-97$

[4] Howard RD.The anomalous mandibular canine.Br.J Orthod.1976;3;117-21

[5] Bruszt P.neurological anomaly associated with extreme malposition of mandibular canine.J oral surg [chic][1958;11;89-90].

[6] Mitchell L. Displacement of a mandibular canine following fracture of the mandible,br dent j.1993;174;417-8

[7] Shapira Y,Mischler W A,kuflinac MM. The displaced mandibular canine.ASDC J Dent child 1982;49;362-364.

[8] Camilleri S,Scerri E;transmigration of mandibular canines and review of literature and report of five cases. Angle orthod $2003 ; 73 ; 753-762$

[9] Aydin U,Yilmaz H.H..Transmigration of Impacted Dento maxilofac Radiol;2003;32;198-200

[10] Mupparapa M.Patterns of intra osseous transmigration and ectopic eruption of mndibular canines; review of literature and report of nine additional cases. Dentomaxillofac Radiol 2002;31:355-60.).

[11] Ando S.AizaeaK.Nakashima T.Sanka Y.Shimbo K.Kiyikawa k. [Transmigration Process of impacted mandibular cuspid.J Nihon univ sch Dent1964;6;66-71; report of nine additional cases]. Dentomaxillofac radiol 2002;31;355-60

[12] Caldwell J B.Neurological anomaly associated with extreme malposition of a mandibular canine.J Oral surg[chic]1955;8;484-7] 


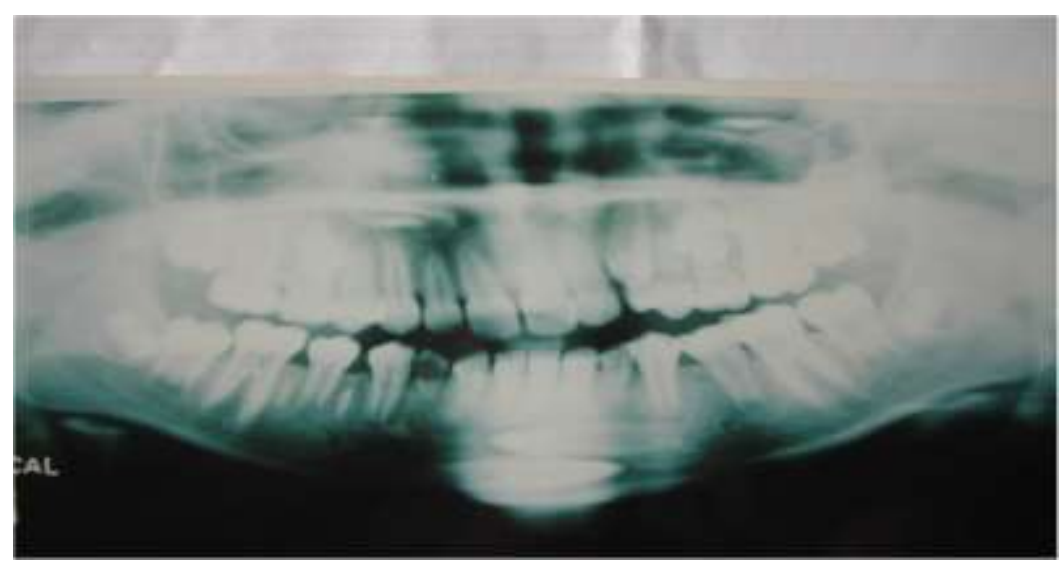

Figure 1 OPG SHOWING BILATERAL KISSING CANINES

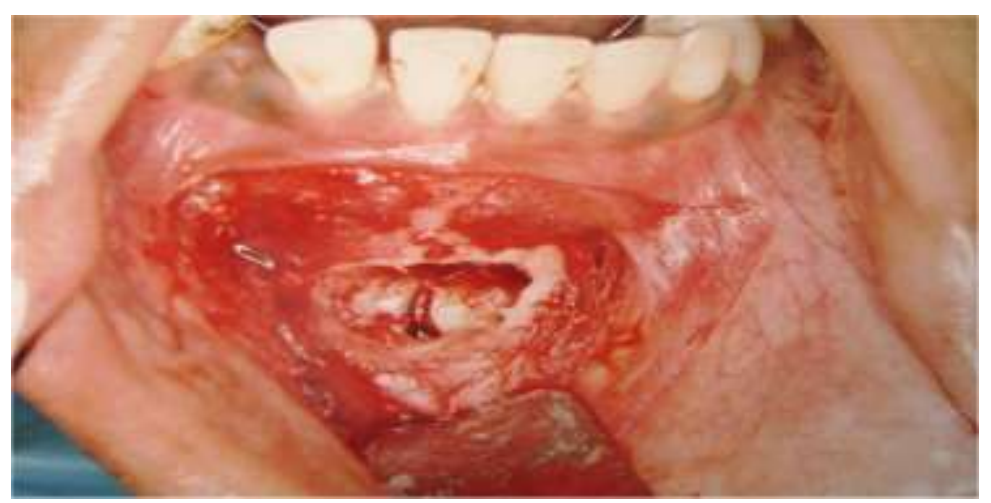

Figure 2DEGLOVING INCISION GIVEN AND TOOTH DECAPITATED

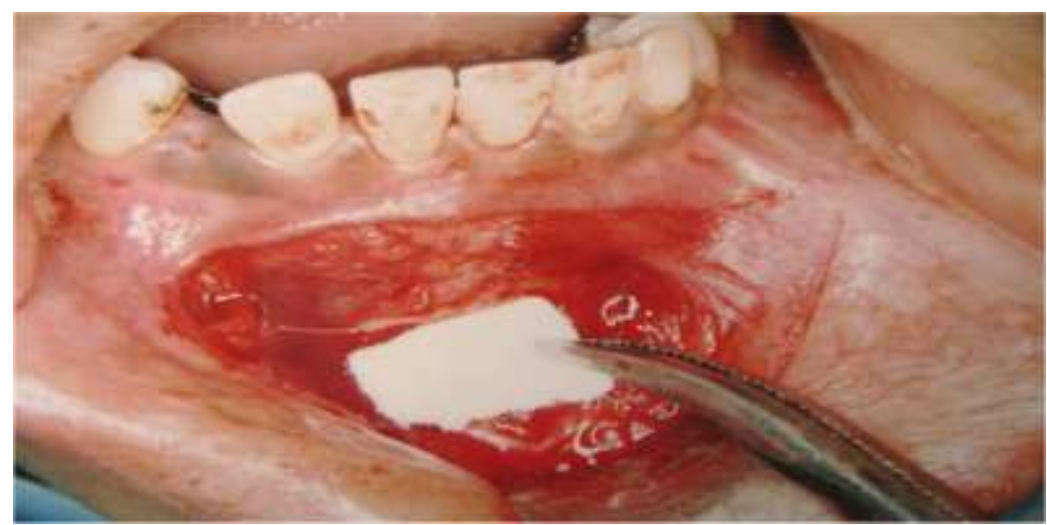

Figure 3 ABGEL BEING PLACED TO COVER THE DEFECT

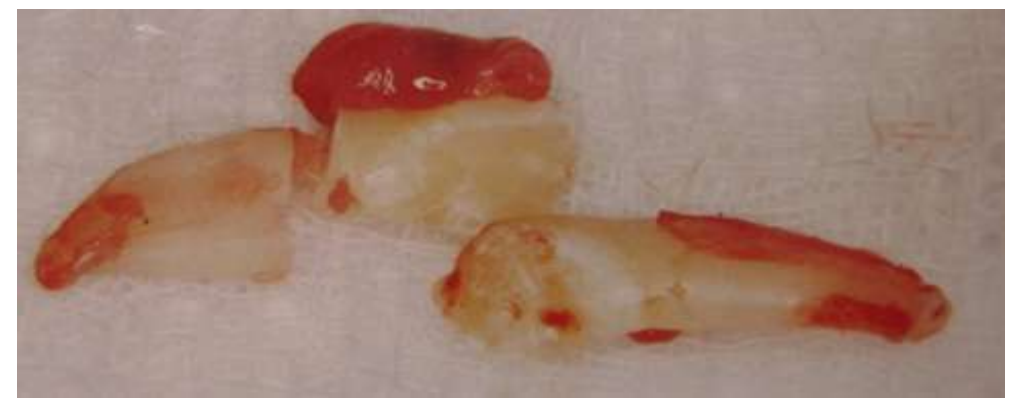

Figure 4 DECAPITATED TOOTH SHOWN 